https://doi.org/10.22319/rmcp.v11i1.5588

Nota de investigación

\title{
Comportamiento productivo y valor nutricional de veza común (Vicia sativa l.) durante otoño-invierno en Zacatecas, México
}

Ricardo A. Sánchez-Gutiérrez ${ }^{\text {a }}$

Juan José Figueroa-Gonzáles a

José Saúl Rivera Vázquez ${ }^{\text {b }}$

Manuel Reveles-Hernández ${ }^{\text {a }}$

Héctor Gutiérrez-Bañuelos ${ }^{b}$

Alejandro Espinoza-Canales ${ }^{\mathrm{c} *}$

${ }^{a}$ Instituto Nacional de Investigaciones Forestales Agrícolas y Pecuarias (INIFAP). Campo Experimental Zacatecas. Calera de V.R., Zacatecas, México.

b Universidad Autónoma de Zacatecas. Unidad Académica de Medicina Veterinaria y Zootecnia. Zacatecas, México.

c Universidad Autónoma de Zacatecas, Unidad Académica de Agronomía. Zacatecas, México.

*Autor de correspondencia: alexespinoza82@live.com.mx

\section{Resumen:}

El momento óptimo de cosecha y la calidad nutricional esperada de forraje de veza común permitiría mejorar su uso en los sistemas de producción. El objetivo fue determinar la producción de forraje, contenido de proteína cruda y los componentes de rendimiento en seis fechas de corte de veza común durante el ciclo otoño-invierno de riego en Zacatecas. El experimento se sembró el 9 de diciembre del 2016 bajo un diseño experimental completamente al azar con cuatro repeticiones. La cosecha de las plantas se realizó cada 14 días, iniciando a los 47 después de la siembra. Las variables medidas fueron: rendimiento de 
forraje verde y seco, producción de hoja verde y senescente, tallo, flor, vaina y proteína cruda. Los datos se analizaron con medidas repetidas mediante el paquete estadístico SAS. En el forraje verde se observó un incremento $(P<0.05)$ desde el día 47 con $493 \mathrm{~kg} \mathrm{ha}^{-1}$ hasta el día 103 con 20,562 $\mathrm{kg} \mathrm{ha}^{-1}$. El forraje seco mostró un incremento constante $(P<0.05)$ desde el día 47 con $14 \mathrm{~kg} \mathrm{MS} \mathrm{ha}^{-1}$ hasta llegar al 103, con 3,796 kg MS ha-1. En proteína cruda se observa un decremento después del día $60(P<0.05)$ y se mantiene similar desde el día 75 hasta el 103 con valores desde 27 hasta $29 \%$ base seca. La acumulación más baja fue al 117 con $20.7 \%$, diferente a los días anteriores $(P<0.05)$. Se concluye que la fecha óptima para el uso en la alimentación del ganado sería alrededor del día 100, al inicio de la floración.

Palabras clave: Veza común, Rendimiento, Proteína cruda.

Recibido: 05/09/2018

Aceptado: 19/02/2019

Los forrajes clasificados de buena calidad proteica que se utilizan para el pastoreo o corte son los de más alta demanda para la alimentación animal. En México existen alrededor de 33.5 millones de bovinos y 17.4 millones de ovinos y caprinos que demandan alimento de calidad para satisfacer las necesidades de mantenimiento y de producción ${ }^{(1)}$. La alfalfa (Medicago sativa L.) es el cultivo más utilizado en los sistemas de producción de carne y leche, sin embargo, una de las principales limitantes que presenta, es la disminución de la producción durante los meses de invierno ${ }^{(2)}$. En el norte-centro de México los cultivos alternativos a la alfalfa para el ciclo otoño-invierno son los cereales de grano pequeño como la avena, cebada, trigo y triticale ${ }^{(3)}$, los cuales se caracterizan por su regular o baja calidad proteica cuando presentan su mayor producción de forraje seco ${ }^{(4,5)}$. No obstante, existen leguminosas forrajeras anuales que se utilizan en los sistemas de producción con fines de hacer un uso más eficiente de los recursos, ya que, se obtiene forraje con alto contenido de proteína cruda, y mejoran las propiedades del suelo ${ }^{(6)}$.

Veza común se utiliza para henificado o pastoreo y tolera bajas temperaturas hasta por debajo de los $-10{ }^{\circ} \mathrm{C}^{(7)}$. Tiene la capacidad de responder a la fijación de nitrógeno con mayor cantidad que algunos cereales de grano pequeño, y sobre todo cuando existen las condiciones restringidas de fertilizantes nitrogenados ${ }^{(8,9)}$. El hábito de crecimiento de esta especie llega a ser trepadora cuando se encuentra en competencia con otro cultivo, por lo que se puede asociar con algún cereal ${ }^{(10)}$. Dhima et $a l^{(11)}$ reportaron un incremento de forraje desde 3 hasta $33 \%$ cuando se mezcla la veza con avena, triticale o cebada. Lithourgidis et al ${ }^{(12)}$ mencionan que la calidad del forraje de la mezcla no presenta mejoría en la fibra detergente neutro y ácido, en comparación con los cereales, pero la proteína cruda disminuye, debido a que la 
veza en monocultivo llega a tener valores desde un 14 hasta $45 \%$. Un estudio relacionado con la respuesta animal en pequeños rumiantes menciona ventajas con el uso de este cultivo, Berhane y Eik ${ }^{(13)}$ utilizaron como suplemento el forraje henificado desde 0 hasta el $1.5 \%$ del peso vivo de cabras en lactancia, y reportaron que a medida que se incrementa el forraje la producción de leche aumenta del 40 al $50 \%$ al finalizar el periodo de lactación. En corderos alimentados con praderas de cebada y veza, ambos en monocultivo más la suplementación de un concentrado, se logró reducir hasta un $20 \%$ en los costos de alimentación con el uso de la veza ${ }^{(14)}$. En Zacatecas, la veza podría ser un cultivo alternativo a las 21,478 ha que se dedican al cultivo de avena durante el ciclo otoño-invierno ${ }^{(1)}$. Sin embargo, se desconoce tanto la producción y calidad del forraje como la etapa o fecha optima de corte. Por tanto, el objetivo del trabajo fue determinar la producción de forraje, contenido de proteína cruda y los componentes de rendimiento en seis fechas de corte de veza común (Vicia sativa L.), con fines para determinar el momento óptimo de cosecha durante el ciclo otoño-invierno de riego en Zacatecas.

El experimento se estableció en el INIFAP-Campo Experimental Zacatecas, ubicado en las coordenadas geográficas de $102^{\circ} 39^{\prime} \mathrm{O}$ y $23^{\circ} 36^{\prime} \mathrm{N}$, a 2,192 msnm. El clima se clasifica como semiárido, la precipitación media anual de $340 \mathrm{~mm}$ y el pico se registra en los meses de julio y agosto, la temperatura media oscila de 12.4 a $21.8{ }^{\circ} \mathrm{C}$ durante los meses de diciembre a mayo ${ }^{(15)}$. Durante el ciclo del cultivo solo se registraron $29.7 \mathrm{~mm}$, lo que representó un $42 \%$ de lo esperado (Figura 1). Asimismo, el suelo es franco arenoso con $\mathrm{pH}$ de 7. El experimento se sembró a tierra venida el 9 de diciembre del 2016 bajo un diseño experimental completamente al azar con cuatro repeticiones. Se instaló un sistema de riego con cintilla superficial y se aplicó una lámina aproximada de $60 \mathrm{~cm}$. La dosis de fertilización fue 60 - 60 Nitrógeno y Fósforo, respectivamente. La siembra se realizó en surcos de $0.76 \mathrm{~m}$ a doble hilera de plantas y la cantidad de semilla fue de $80 \mathrm{~kg} \mathrm{ha}^{-1}$ (90\% de viabilidad). La unidad experimental fue de ocho surcos de $0.76 \mathrm{~m}$ de ancho por $5.00 \mathrm{~m}$ de largo, y la parcela útil fueron dos surcos de $5 \mathrm{~m}$ de largo por corte, se omitieron los dos surcos de la orilla de la unidad experimental. La cosecha de las plantas se realizó cada 14 días, iniciando a los 47 días después de la siembra (DDS). 
Figura 1: Precipitación mensual acumulada $(\mathrm{mm})$ y temperatura media $\left({ }^{0} \mathrm{C}\right)$ del INIFAPCampo Experimental Zacatecas en el año 2016

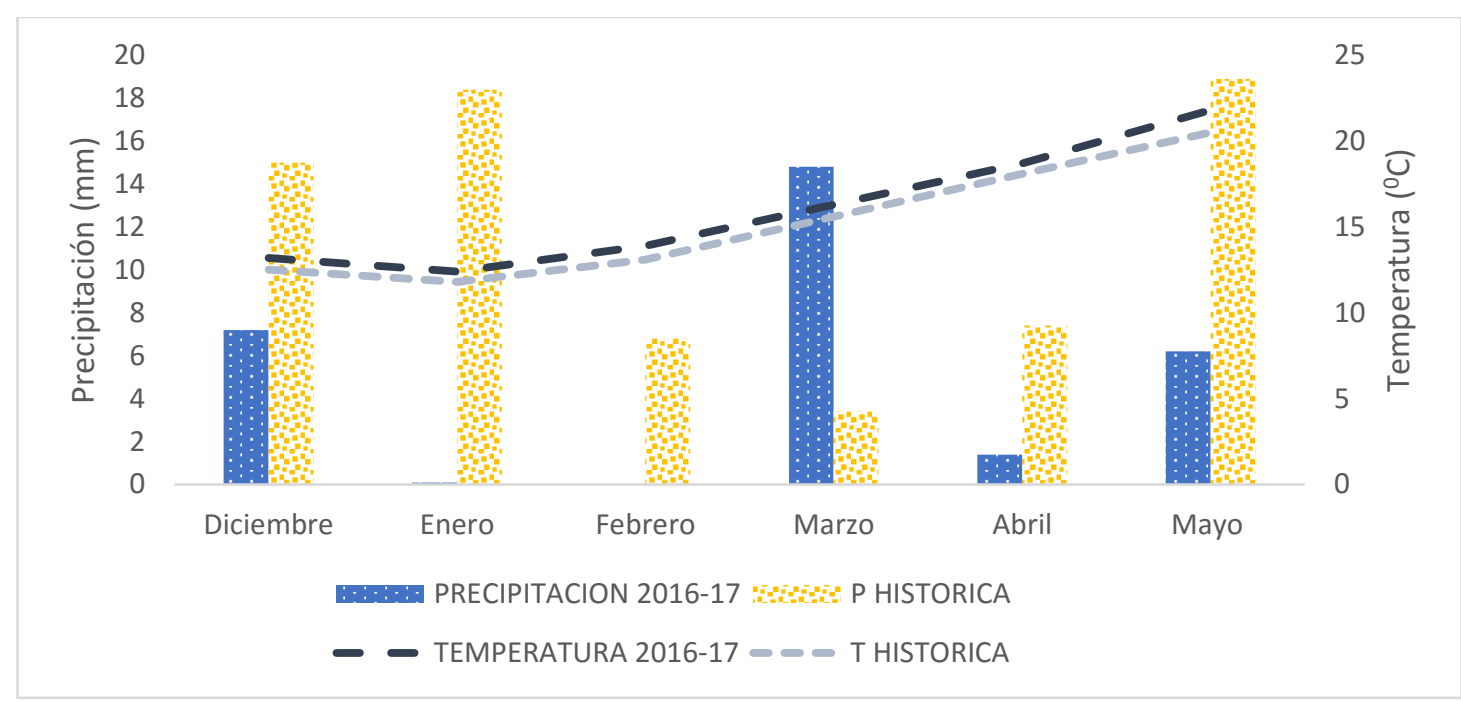

Las variables medidas fueron: rendimiento de forraje verde (FV) y seco (FS), producción de los componentes de rendimiento de la materia seca; hoja verde (HJV) y senescente (HJS), tallo (T), flor (F), vaina $(\mathrm{V})$ y proteína cruda (PC) de la planta completa por fecha de corte. La estimación del forraje verde fue a partir de la biomasa que se cortó de la parcela útil a 5 $\mathrm{cm}$ del suelo y se pesó. Posteriormente, se tomaron dos muestras al azar de $0.5 \mathrm{~kg}$, una de ellas se separó y pesaron las partes vegetativas de la planta, tallo, hoja verde y senescente, flor y vaina, y la otra fue usada en la determinación de proteína. Después, todas las partes vegetativas y la muestra restante fueron llevadas a una estufa a $55{ }^{\circ} \mathrm{C}$ durante $48 \mathrm{~h}$. Por último, se pesaron las muestras y se determinó el porcentaje de materia seca. Con la producción de forraje verde y el porcentaje de materia seca se estimó el rendimiento de forraje seco por hectárea. Las muestras secas con todos los componentes se procesaron en un molino tipo Willy con una criba de un milímetro. El nitrógeno total con el cual se calculó la PC fue mediante el análisis de combustión de Dumas ${ }^{(16)}$.

Los datos se evaluaron mediante un análisis estadístico para un diseño de completamente al azar con medidas repetidas, mediante el procedimiento "PROC MIXED" del paquete estadístico de SAS ${ }^{(17)}$. La comparación de medias se realizó mediante Lsmeans y se eligió una probabilidad menor al $5 \%{ }^{(17)}$. Se realizó un análisis de regresión en forraje verde, seco y proteína cruda con la finalidad de observar la tendencia.

En la Figura 2 se presenta la dinámica de crecimiento del forraje verde y seco de la veza común bajo condiciones de riego en el ciclo otoño-invierno 2016-2017 en Zacatecas. En el forraje verde se observó un incremento significativo desde el día 47 con $493 \mathrm{~kg} \mathrm{FV} \mathrm{ha}^{-1}$ hasta el día 103 con 20,562 $\mathrm{kg} \mathrm{ha}^{-1}$ que fue cuando presentó el pico más alto de producción disminuyendo en los siguientes muestreos $(P<0.05)$. El mejor modelo ajustado fue un 
polinomial de tercer grado, debido a que el coeficiente de determinación fue alto $\left(\mathrm{R}^{2}=0.904\right)$. La dinámica de crecimiento de forraje seco mostró un incremento constante $(P<0.05)$ desde el día 47 con $14 \mathrm{~kg} \mathrm{MS} \mathrm{ha}^{-1}$ hasta llegar al 103, donde presentó 3,796 $\mathrm{kg} \mathrm{MS} \mathrm{ha}^{-1}$, días después la producción se mantuvo similar $(P>0.05)$. El modelo polinomial de tercer grado explicó el $96.3 \%\left(\mathrm{R}^{2}=0.963\right)$ de la variabilidad total de los datos. Los resultados de forraje verde coinciden con los mencionados por Lithoutgidis et $a l^{(18)}$ puesto que reportaron $20.49 \mathrm{t}$ MS $\mathrm{ha}^{-1}$ en el pico alto de producción y un comportamiento cuadrático. Los rendimientos de forraje seco se asemejan a la producción obtenida en Zacatecas donde obtuvieron de 2.6 a $4.2 \mathrm{n} \mathrm{MS} \mathrm{ha}^{-1}$ en cuatro fechas de cosecha, 85, 92, 106 y 118 días después de la siembra ${ }^{(19)}$, sin embargo, los autores no mencionan diferencias estadísticas entre fechas. La información generada con la dinámica de crecimiento de la veza común en otoño-invierno pudiera ser una herramienta en la toma de decisiones para los diferentes usos de este cultivo. En trabajos de investigación se recomienda la veza como una alternativa para la conservación del suelo, ya que lo protege de la erosión y a la vez incrementa la materia orgánica ${ }^{(20,21)}$. Por lo tanto, la fecha óptima para la utilización de abono verde sería del día 100 al 110 después de la siembra, en vista que es cuando hay suficiente biomasa con la mayor cantidad de agua en el follaje. Lo anterior, sería una condición favorable para que los microorganismos del suelo descompongan y mineralicen la materia orgánica ${ }^{(22)}$.

Figura 2: Dinámica de crecimiento del forraje verde y seco de veza común durante el ciclo otoño-invierno en Zacatecas 2016-2017

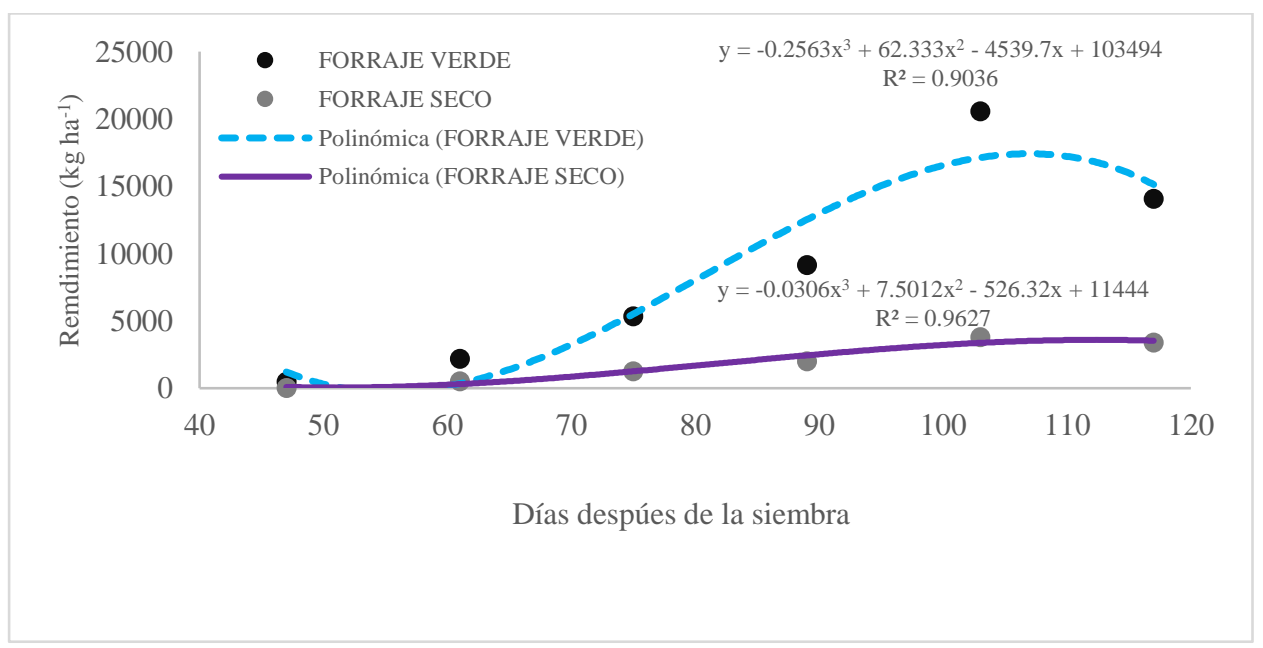

En la Figura 3 se presenta la acumulación de proteína cruda y la producción de forraje seco de veza común (Vicia sativa) bajo condiciones de riego en el ciclo otoño-invierno 2016-2017 en Zacatecas. Se observa un decremento de la proteína cruda después del día $60(P<0.05)$ y se mantiene similar desde el día 75 hasta el 103 con valores desde 27 hasta $29 \%$. La acumulación más baja fue al 117 con $20.7 \%$, diferente a los días anteriores $(P<0.05)$. El 
modelo polinomial de tercer grado fue el que mejor se ajustó con un coeficiente de determinación $\left(\mathrm{R}^{2}\right)$ de 0.99 . En veza común la proteína cruda decrece conforme el ciclo finaliza $^{(23)}$, los rangos reportados de floración hasta casi madurez fisiológica fueron desde 32 hasta $14.4 \%$. Igualmente, en Zacatecas del día 85 al 118 se obtuvieron rangos del 29 al $24 \%$ de PC, sin presentar diferencias estadísticas ${ }^{(19)}$. Uno de los factores principales que afecta la producción animal es la calidad de los alimentos, en forrajes la calidad no solo está ligada al estadio de desarrollo de la panta, sino también a la especie y a la adaptación al medio ambiente. Por lo tanto, para el ciclo otoño-invierno en Zacatecas la fecha óptima de corte sería alrededor del día 100, después el forraje seco se mantiene, pero se disminuye el contenido de la proteína cruda.

Figura 3: Acumulación de proteína cruda (PC) y producción de forraje seco (FS) de veza común durante el ciclo otoño-invierno en Zacatecas 2016-2017

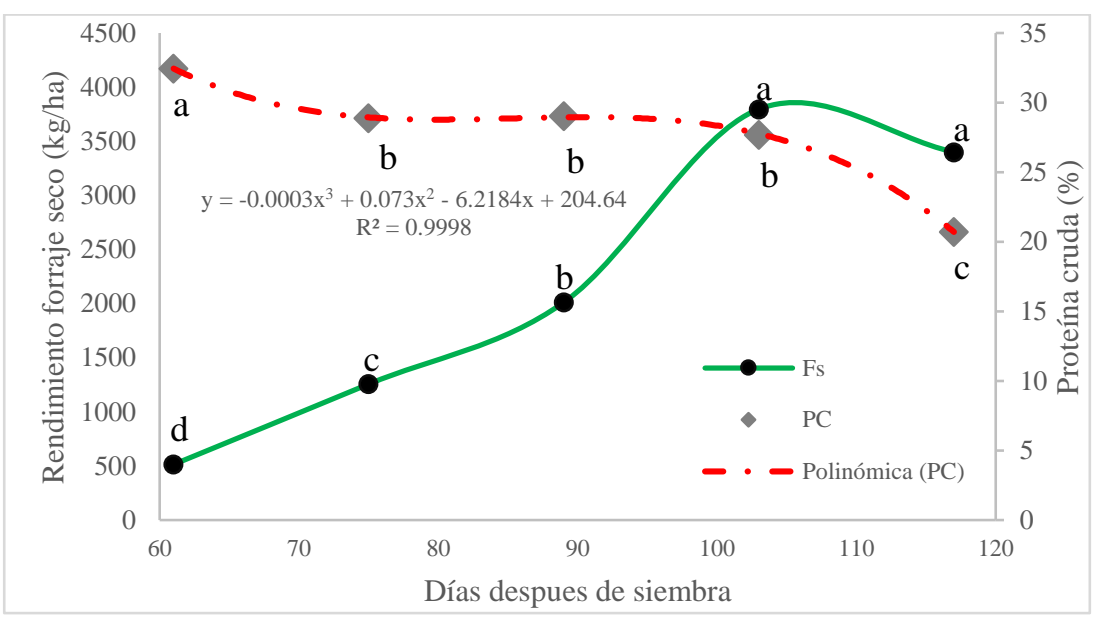

En el Cuadro 1 se presenta la producción de materia seca de los componentes morfológicos de veza común (Vicia sativa) durante el ciclo otoño-invierno del 2016-2017 en Zacatecas. En el componente de tallos se presenta un crecimiento significativo a partir del día 47 con 26 kg MS ha ${ }^{-1}$ hasta el día 89 , después se mantiene constante $(P>0.05)$ hasta el día 117 con producciones desde 912 hasta 1,091 kg MS ha-1 ${ }^{-1}$ La producción de hojas verdes comenzó un incremento $(P<0.05)$ desde el día 47 con $30 \mathrm{~kg} \mathrm{MS} \mathrm{ha}^{-1}$ hasta el día 103 con $1,487 \mathrm{~kg}$ MS $\mathrm{ha}^{-1}$, después la producción fue similar. La hoja senescente y la flor se empezaron a registrar a partir del día 103, las producciones fueron similares $(\mathrm{P}>0.05)$ entre estos dos últimos cortes, de 661 hasta 1,255 y de 871 hasta172 $\mathrm{kg} \mathrm{MS} \mathrm{ha}^{-1}$, respectivamente. La producción de vaina se observó en el último corte con $164 \mathrm{~kg} \mathrm{MS} \mathrm{ha-1}$. Por otro lado, la producción de hoja verde 
se mantuvo por encima de tallos, lo cual es un buen indicador de la calidad y consumo de forraje, ya que allí es donde se acumula la mayor cantidad de proteína cruda y es la parte más digestible de la planta ${ }^{(24)}$. A partir del día 103 la veza presentó hojas secas o amarillentas, síntomas que indica el inicio del estadio de desarrollo de la senescencia, proceso en el cual la planta distribuye los nutrientes que se encuentran en dichas hojas para desarrollar la floración y el llenado de grano ${ }^{(25)}$.

Cuadro 1: Producción de los componentes de veza común (Vicia sativa) en base seca y verde $( \pm \mathrm{DE})$ en seis cortes durante el ciclo otoño-invierno en Zacatecas, 2016-2017

\begin{tabular}{|c|c|c|c|c|c|}
\hline DDS & $\begin{array}{l}\text { TLL } \\
\left(\mathrm{kg} \mathrm{ha}^{-1}\right)\end{array}$ & $\begin{array}{l}\text { HJV } \\
\left(\mathrm{kg} \mathrm{ha}^{-1}\right)\end{array}$ & 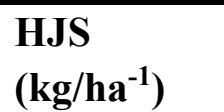 & $\begin{array}{l}\text { FR } \\
\left(\mathrm{kg} \mathrm{ha}^{-1}\right)\end{array}$ & $\begin{array}{l}\mathrm{V} \\
\left(\mathrm{kg} \mathrm{ha}^{-1}\right)\end{array}$ \\
\hline 47 & $27 \pm 4 \mathrm{a}$ & $30 \pm 2 \mathrm{~d}$ & & & \\
\hline 61 & $229 \pm 102 b$ & $285 \pm 122 \mathrm{c}$ & & & \\
\hline 75 & $557 \pm 55 \mathrm{c}$ & $699 \pm 99 \mathrm{bc}$ & & & \\
\hline 89 & $913 \pm 194 \mathrm{~d}$ & $1098 \pm 474 \mathrm{ab}$ & & & \\
\hline 103 & $1085 \pm 186 \mathrm{~d}$ & $1488 \pm 348 \mathrm{a}$ & $662 \pm 469 \mathrm{a}$ & $871 \pm 194 \mathrm{a}$ & \\
\hline 117 & $1091 \pm 228 \mathrm{~d}$ & $1183 \pm 570 \mathrm{a}$ & $1256 \pm 425 \mathrm{a}$ & $172 \pm 64 \mathrm{a}$ & $164 \pm 40$ \\
\hline
\end{tabular}

DDS= días después de la siembra; TLL= tallo verde; HJV= hoja verde; HJS= hoja senescente; FR= flor; V= vaina; $\mathrm{DE}=$ desviación estándar.

En el presente estudio se determinó que la fecha óptima para realizar el corte es alrededor del día 100, a partir de este día la planta presenta sus componentes de floración, por lo tanto, coincide con algunos autores donde realizaron trabajos de investigación y realizaron el corte en un estadio de la planta de $20 \%$ de floración ${ }^{(10,11,26)}$. Debido a que la veza común tiene las características de ser un componente para el desarrollo de una producción animal sustentable, se recomienda continuar con un programa de selección o mejoramiento genético, puesto que Flores $^{(27)}$ reportó en Zacatecas seis líneas superiores en rendimiento a la veza que se comercializa en México. Por lo tanto, es la importancia de la identificación de líneas con potencial forrajero en monocultivo, con hábitos de crecimiento erecto o semi-erecto las cuales se pudieran mezclar con cereales de grano pequeño.

De acuerdo a la dinámica de crecimiento de la veza común, la fecha óptima para la utilización como abono verde sería alrededor del día 100, la cual coincide con el corte para el uso en la alimentación del ganado, ya que se tiene el mejor rendimiento con buena cantidad de proteína cruda. Además, siendo otra alternativa para realizar el corte y lograr el mejor aprovechamiento de la veza cuando inicia el estadio de floración. 


\section{Literatura citada:}

1. SIAP. Sistema de Información Agroalimentario y Pesquero. SAGARPA, México. https://www.gob.mx/siap. Consultado 12 Sep, 2016.

2. Moreno DL, García AD, Faz CR. Manejo del riego en alfalfa. Producción y utilización de la alfalfa en la zona norte de México. Secretaría de Agricultura, Ganadería y Desarrollo Rural. Instituto Nacional de Investigaciones Forestales y Agropecuarias. Centro de Investigación Regional Norte Centro. Campo Experimental La Laguna; Libro Técnico núm. 2, 2002.

3. Cruz CJJ, Núñez G, Faz R, Reta DG, Serrato HA. Potencial forrajero y eficiencia de uso del agua de canola (Brassica napus L.) en comparación con cultivos tradicionales en el ciclo de invierno. AGROFAZ 2012;12:125-130.

4. Feyissa F, Tolera A, Melaku S. Effects of variety and growth stage on proportion of different morphological fractions in oats (Avena sativa L.). Degefa T, Feyyissa F editors. Proc $15^{\text {th }}$ Ann Conf Ethiopian Soc Anim Prod (ESAP) Ethiopia 2007:47-61.

5. Hadjipanayiotou M, Antoniou I, Theodoridou M, Photiou A. In situ degradability of forages cut at different stages of growth. Livest Prod Sci 1996;45(1):49-53.

6. Kuusela E, Khalili H, Nykänen-Kurki P. Fertilisation, seed mixtures and supplementary feeding for annual legume-grass-cereal pastures in organic milk production systems. Livest Prod Sci 2004;85(2-3):113-127.

7. Mikić A, Mihailović V, Ćupina B, Đorđević V, Milić, D, Duc, G, et al. Achievements in breeding autumn-sown annual legumes for temperate regions with emphasis on the continental Balkans. Euphytica Int J Plant Breed 2011;1(1):5.

8. Ruffo M, Parsons A. Cultivos de cobertura en sistemas agrícolas. Informaciones Agronómicas del Cono Sur 2004-21:13-16.

9. Kurdali F, Sharabi NE, Arslan A. Rainfed vetch-barley mixed cropping in the Syrian semiarid conditions. Plant Soil 1996;183(1):137-148.

10. Caballero R, Goicoechea EL, Hernaiz PJ. Forage yields and quality of common vetch and oat sown at varying seeding ratios and seeding rates of vetch. Field Crop Res 1995;41(2):135-140.

11. Dhima KV, Lithourgidis AS, Vasilakoglou IB, Dordas CA. Competition indices of common vetch and cereal intercrops in two seeding ratio. Field Crop Res 2007;100(23):249-256. 
12. Lithourgidis AS, Dhima KV, Vasilakoglou IB, Dordas CA, Yiakoulaki MD. Sustainable production of barley and wheat by intercropping common vetch. Agron Sustain Dev 2007;27(2):95-99.

13. Berhane G, Eik LO. Effect of vetch (Vicia sativa) hay supplementation to Begait and Abergelle goats in northern Ethiopia: II. Reproduction and growth rate. Small Ruminant Res 2006;64(3):233-240.

14. Rihawi S, Iñiguez L, Knaus WF, Zaklouta M, Wurzinger, M, Soelkner J, Bomfim MAD. Fattening performance of lambs of different Awassi genotypes, fed under cost-reducing diets and contrasting housing conditions. Small Ruminant Res 2010;94(1-3):38-44.

15. Medina GG, Ruiz CA. Estadísticas climatológicas básicas del estado de Zacatecas (Periodo 1961-2003). 1ª ed. México: INIFAP; 2004.

16. AOAC, Official Methods of Analysis.16th ed, Association of Official Analytical Chemists, Gaithersburg, MD, USA. 1999.

17. SAS. SAS/STAT User’s Guide. Statistical Analysis System. Inc. Cary, NC. Versión 9.1. 2011.

18. Lithourgidis AS, Vasilakoglou IB, Dhima KV, Dordas CA, Yiakoulaki MD. Forage yield and quality of common vetch mixtures with oat and triticale in two seeding ratios. Field Crop Res 2006;99(2-3):106-113.

19. Flores-Nájera MDJ, Sánchez-Gutiérrez RA, Echavarría-Cháirez FG, Gutiérrez-Luna R, Rosales-Nieto CA, Salinas-González H. Producción y calidad de forraje en mezclas de veza común con cebada, avena y triticale en cuatro etapas fenológicas. Rev Mex Cienc Pecu 2016;7(3):275-291.

20. Navarro-Garza H, Olvera P, Antonia M, Castillo-González F. Evaluación de cinco especies vegetales como cultivos de cobertura en valles altos de México. Rev Fit Mex 2007;30(2):151-157.

21. Viteri S, Martínez J, Bermúdez A. Selección de abonos verdes para los suelos de Turmequé (Boyacá). Agron Colom 2008;26(2):332-339.

22. Celaya-Michel H, Castellanos-Villegas AE. Mineralización de nitrógeno en el suelo de zonas áridas y semiáridas. Terra Latinoamericana 2011;29(3):343-356.

23. Ramos- Morales E, Sanz- Sampelayo MR, Molina- Alcaide E. Nutritive evaluation of legume seeds for ruminant feeding. J Anim Physiol Anim Nutr 2010-94(1):55-64.

24. Alatorre-Hernández A, Guerrero-Rodríguez J, Olvera-Hernández JI, Aceves-Ruíz E, Vaquera-Huerta H, López SV. Productividad, características fisicoquímicas y 
digestibilidad in vitro de leguminosas forrajeras en trópico seco de México. Rev Mex Cienc Pecu 2018;9(2):296-315.

25. Buchanan-Wollaston V, Earl S, Harrison E, Mathas E, Navabpour S, Page T, Pink D. The molecular analysis of leaf senescence-a genomics approach. Plant Biotechnol J 2003;1:3-22.

26. Erol A, Kaplan M, Kizilsimsek M. Oats (Avena sativa) - common vetch (Vicia sativa) mixtures grown on a low-input basis for a sustainable agriculture. Tropical Grasslands 2009;43:191-196.

27. Flores OMA, Gutiérrez LR, Palomo RM. Veza común y Lathyrus sativus L.: alternativas para producir forraje en Zacatecas. Instituto Nacional de Investigaciones Forestales y Agropecuarias. Folleto científico No.13. 2007. 